\title{
A Feasibility Study of a Noncontact Torque Sensor with Multiple Hall Sensors
}

\author{
Kyungshik Lee and Chongdu Cho \\ Department of Mechanical Engineering, Inha University Graduate School, 100 Inha-ro, Nam-Gu, Incheon 402-751, Republic of Korea \\ Correspondence should be addressed to Chongdu Cho; cdcho@inha.ac.kr
}

Received 29 October 2014; Revised 16 December 2014; Accepted 29 December 2014

Academic Editor: Luisa Torsi

Copyright (c) 2015 K. Lee and C. Cho. This is an open access article distributed under the Creative Commons Attribution License, which permits unrestricted use, distribution, and reproduction in any medium, provided the original work is properly cited.

\begin{abstract}
The feasibility of a noncontact sensor is investigated. This type of sensor can potentially be used for torque measurement in a speedvariable power transmission system. Torque can be read by examining the phase difference between two induction signals from respective magnetic sensors that detect the magnetic field intensity of permanent magnets mounted on the surface of a shaft in rotation. A real-time measuring algorithm that includes filtering and calibration is adopted to measure the torque magnitude. It is shown that this new torque sensor can perform well under rotation speeds ranging from $300 \mathrm{rpm}$ to $500 \mathrm{rpm}$. As an interim report rather than a complete development, this work demonstrates the feasibility of noncontact torque measurement by monitoring a magnetic field. The result shows an error of less than $2 \%$ within the full test range, which is a sufficient competitive performance for commercial sensors. The price is very low compared to competitors in the marketplace, and the device does not require special handling of the shaft of the surface.
\end{abstract}

\section{Introduction}

Torque is an axially twisting moment that is measured on the shafts of rotating machines. For example, in automobiles, electric motors, and helicopters, the technique of torque feedback control is needed to reduce fuel consumption and improve ride comfort. Nonetheless, no torque measurement solution has proven to be reliable and repeatable for successful commercialization in the marketplace. This holds true especially in the automotive industry, which seeks to achieve a low-cost and in situ dynamic torque control without major change of existing designs. The installation method faces great difficulty because it requires reliable and available strain-gauge-type torque sensors in automobiles. For example, common strain-gauge-type sensors must be inserted into the middle of the power transmission shaft using a slip ring, which requires critical maintenance. Another difficulty is the necessity of keeping performance at a high oil temperature of approximately $200^{\circ} \mathrm{C}$. The strain-gauge-type sensor is normally reliable only under $80^{\circ} \mathrm{C}$, and it is difficult to seal against hot oil.

To resolve these difficulties, studies [1] have pursued the development of a high-performance torque sensor with a reasonable price. Among noncontact-type sensors, a magnetostrictive-type torque sensor uses one of the potential techniques which is based on the iteration of the periodic time difference [2-4]. Contactless technique sensors can be used with fewer shaft design changes. The elastomagnetic effect of ferromagnetic materials exhibits the phenomenon that strain or stress induced by the torque on the shaft can change the magnetic properties of a magnetostrictive material and cause hysteresis deformation and permeability [5]. A frequency modulation- (FM-) type wireless CMOS IC magnetoimpedance (MI) sensor is constructed using flashannealed amorphous wire [6]. A number of resonant wireless surface acoustic wave (SAW) sensors have been developed for measuring temperature, pressure, and torque in various automotive applications [7]. An embedded capacitive grating torque sensor and measuring system have been developed [8]. The capacitive torque sensor is composed of two capacitive displacement sensors in order to measure a twist angle [9].

Moving magnet technologies (MMT) and the SAW-type sensor are used successfully in the current market, but the installation of these items requires extensive modification of the target shaft. The best advantage of the new methods is that there are no critical design changes to the current system. 


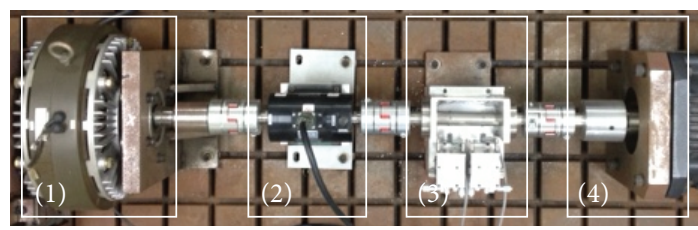

Figure 1: Photo of test bench.

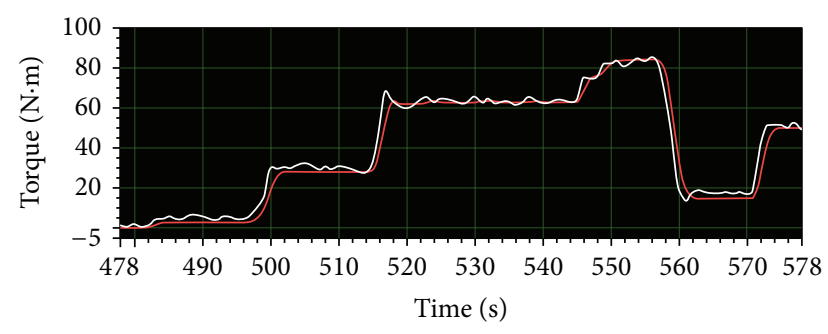

FIGURE 2: Front screenshot of torque sensor program.

For example, MMT can measure the shaft angle between two rotating shafts that are linked by a torsion bar [10], but our sensor directly measures the distortion angle of a shaft.

This paper proposes a new sensor concept and measurement principle using (1), where the shaft twist angle in a linear elastic state is proportional to the net in situ torque of a prismatic circular-shaped shaft. When a shaft has a radius $R$ and a length $L$ and the shear modulus of the shaft material is $G$, torque $T$ has a relation with a twisting angle $\Phi$ in rad:

$$
T=\frac{\pi R^{4} G}{2 L} \Phi .
$$

On the other hand, a traditional optical sensor may be used to measure a reflected distance using a specially processed surface of the axis to detect a twist angle. However, this method is limited in some special environments. In this study, we propose a new torque sensor in which multiple permanent magnet pairs make the sensor applicable even in harsh and hot environments such as oil and dust in working machines. Increasing the longitudinal separation $L$ of the two Hall sensors improves the accuracy of measurements. However, a major weak point is that not every target system has sufficient space to do so.

\section{Measurement System Configuration}

2.1. Hardware Configuration. A test bench, which is detailed in Figure 1, is produced to evaluate the feasibility of the newly proposed torque sensor. The bench consists of (1) a friction powder clutch, (2) commercial inline torque sensors for comparison purposes, (3) the torque sensor under development in this study, and (4) a servomotor that connects the shafts, couplings, data acquisition (DAQ) card, and a personal computer.

For analysis and visual results, we used the LabVIEW program, as shown in Figure 2. The red line indicates the data from the inline-type sensor, and the white line is from the new sensor. The dialogue shows that the compared data

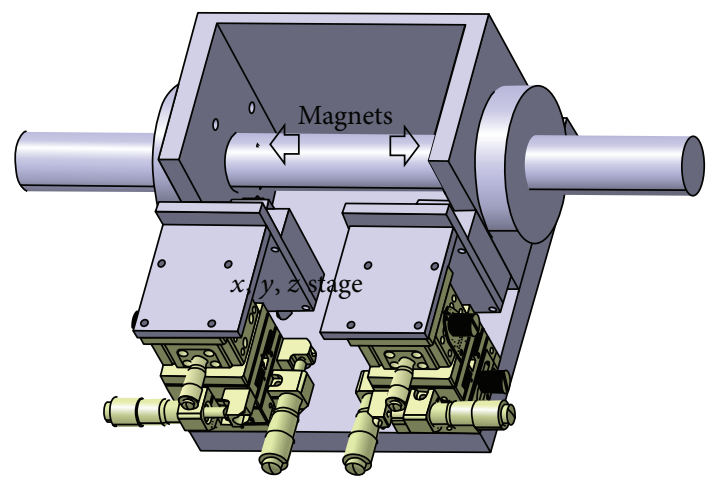

FIGURE 3: Schematic of torque sensor bench and shaft.

from the strain-gauge-type sensor can be used as evaluation criteria and to help develop the new sensor.

A Hall sensor is a device that proportionally converts a recognized magnetic field strength to DC voltage, which is typically used for position recognition and detecting rotation speed. Aichi Steel's AMI302 sensor has high sensitivity and low magnetic field strength (full scale 2 Gauss) and can measure a complex three-axis magnetic field. The AMI302 is not capable of measuring the magnetic field in the three axis directions at the same time. Measuring in the $x, y$, and $z$ directions can be selected by a digital signal.

In order to change the distance between the four pairs of magnets and a pair of AMI302 magnetic sensors, a precision bench was fabricated with a $3 \mathrm{D}$ adjustable stage, as shown in Figure 3. An optimum gap between the sensor and the magnet was obtained from the results of experiments. In the experimental results, the optimum gap was determined by the position that measured magnetic field strength within the range of the full scale of the sensor to detect clear signals.

A servomotor (Mitsubishi Electric Corporation MR-J2S$12 \mathrm{KA}$, with a maximum torque of $200 \mathrm{~N} \cdot \mathrm{m}$ ) is used in this study for dynamic testing to apply the desired rotational speed according to an external analog control signal provided by the software. To remove the noise generated by the highcapacity servomotor, ferrite cores and noise-shielding cable were employed. A powder clutch to brake the torsional shaft was mounted on the opposite side of the motor.

The proposed commercial sensor (Haiam HKT-TRC strain-gauge type) is installed and acts as a reference to compare the torque. This sensor has a real-time feedback function and can measure torque up to $200 \mathrm{~N} \cdot \mathrm{m}$, as well as important specific nonlinearities. The sensor's hysteresis and repeatability of $\pm 0.5 \%$ make it a good candidate for a precise comparison.

2.2. Magnet Selection. A neodymium magnet ( $2 \mathrm{~mm}$ hexagon size) is designed as a test magnet. The rubber magnet was used in previous experiments [11-13] and did not exhibit good repeatability. This problem is believed to be a result of a weak magnetic field, high sensitivity to electrical noise generated by the servomotor and various test equipment, and the magnet's nonlinear deformation characteristics. 
The neodymium magnet, a widely used type of rareearth magnet, is a permanent magnet made from an alloy of neodymium, iron, and boron to form the $\mathrm{Nd}_{2} \mathrm{Fe}_{14} \mathrm{~B}$ tetragonal crystalline structure [14]. The most outstanding characteristic of the neodymium magnet is related to temperature: energy loss in the power transmission shaft is mainly dissipated as heat energy. The Curie temperature of the magnet is $310-370^{\circ} \mathrm{C}$, and its maximum operating temperature is $80-$ $200^{\circ} \mathrm{C}$. The density of the magnet is $7.4-7.6 \mathrm{~g} / \mathrm{cm}^{3}$, so the magnets in the test are just $7.4 \mathrm{mg}$ each. Those weights can be ignored by the dynamic unbalanced mass on the shaft.

\section{Signal Processing and Algorithm}

3.1. Noise-Filtering Method. When the neodymium magnet is set at a $12 \mathrm{~mm}$ gap from the sensor, the maximum value of the measured magnetic field reaches a sufficient value of 2 Gauss. The noise and Hall-effect (HE) sensor outputsignal contrast ratio are reduced to $10 \%$, which is helpful in improving precision.

Nevertheless, many large-capacity electrical components, such as the servomotor, can generate considerable highfrequency noise. In order to remove this noise, a low-pass filter with a $60 \mathrm{~Hz}$ or higher cutoff frequency is applied by software. The major signal frequency is approximately $33 \mathrm{~Hz}$ at $500 \mathrm{rpm}$. To increase the reliability of the data, a higher cutoff frequency is useful at $500 \mathrm{rpm}$ or higher. If a new measurement system is applied to an internal combustion engine (as in the transportation industry) and reduces the distance between the HE sensor and DAQ board, the signal would be much clearer, and the cutoff frequency of the lowpass filter (LPF) could be increased.

A spline fitting [15] and averaging filter [16] are applied through LabVIEW to obtain a smooth curve in which to detect peaks of the signal.

3.2. Theoretical and Experimental Formula. The concept of this experiment is to develop a detection method of corresponding individual peaks of magnetic field strength waves to estimate a twisted angle. Therefore, detecting peaks from a signal acquisition is the most important factor. Based on a discrete data set, a best-fit spline wave is determined by the $n$-dimensional spline fitting process data, from which a peak can be estimated.

The correlation between torque and twist angle can be expressed in the following equation. Equation (2) is a modified version of (1) and can be applied to four magnets that are configured in pairs where the measured time is $\Delta t_{i}$ and the initial time is $\Delta t_{i}^{\prime}$ :

$$
\begin{aligned}
T & =\frac{n \pi^{2} R^{4} G}{60 L} \frac{1}{4} \sum_{i=1}^{4}\left(\Delta t_{i}-\Delta t_{i}^{\prime}\right) \\
& =\frac{n \pi^{2} R^{4} G}{60 L} \Delta t_{\text {mean }}=C \Delta t_{\text {mean }} .
\end{aligned}
$$

$\Delta t_{\text {mean }}$ is checked from the data as a unique parameter of torque measurement, if rotational speed $n$ in $\mathrm{rad} / \mathrm{sec}$ is fixed.
The shear modulus is assumed to be constant because the temperature gap is smaller than $10^{\circ} \mathrm{C}$ in this study.

The constant value $C$ in (2) can be calculated easily as $1.9 \times 10^{6}$ at $400 \mathrm{rpm}$ and $14.0 \times 10^{6}$ at $3000 \mathrm{rpm}$. To maintain a calculated torque value of one sampling period less than $1 \mathrm{~N} \cdot \mathrm{m}$, the system should be designed with sensors and circuits that have sampling frequencies greater than the calculated constant value. A DAQ card is used in experiments with a maximum sampling frequency of $102.4 \mathrm{kHz}$. In order to compensate for the problem of higher data acquisition frequency, the calculation takes the average data of the past few periods and fits the curve.

\section{Results and Discussion}

The four magnet pairs are mounted on the shaft at $90^{\circ}$ increments to compensate for the dynamic reaction during rotation. The effect of pairing the magnets and the calibration data are analyzed in this chapter. The experiments can be classified into three steps.

The first step is calibration to obtain two critical parameters by measuring an initial angle difference between each pair of magnets and the twist angle per applied unit torque (in $\mathrm{rad} / \mathrm{N} \cdot \mathrm{m}$ ). In this step, the role of the commercial sensor is to act as a calibrator.

The second step is the repeated data correction of the first step to check long-term variation and repeatability.

The last step involves comparing the respective data of the commercial sensor and the calculated data from the proposed sensor while changing the rotation speed and applied torque. This step confirms the repeatability and reproducibility of the new suggested measurement method as a torque meter.

4.1. Repeatability of the Initial Interval Measurement Test. A pair of sensors and four pairs of magnets are rarely aligned in an axial position with a no-load condition because it is impossible to install them on an exact axial line. Therefore, one of the important tasks of sensor calibration is to read the initial deviation of each magnet pair at a no-load or specified torque condition. In order to confirm repeatability and reliability as a torque measurement sensor, this parameter has no variation in the short or long term.

As with the experimental results in Figure 4, the initial interval measure is highly irregular at $200 \mathrm{rpm}$. We believe this results from the mechanical gap of the ball bearings and the misalignment of the entire test system. A system that measures torque to provide feedback for a closed-loop system must have a highly precious mechanical balance.

The data of $300 \mathrm{rpm}$ were compared in Figure 5. There is a nonlinear region that has unexpected errors in the lowtorque range. This may occur because the magnets were attached using epoxy, an adhesive material that may not deform linearly or uniformly at the beginning of the twisting of the shaft.

In order to consider removing the effect of temperature, a magnet was mounted with AXIA super glue, which can withstand temperatures up to $120^{\circ} \mathrm{C}$ without changing its physical properties. The variable dependent on temperature 


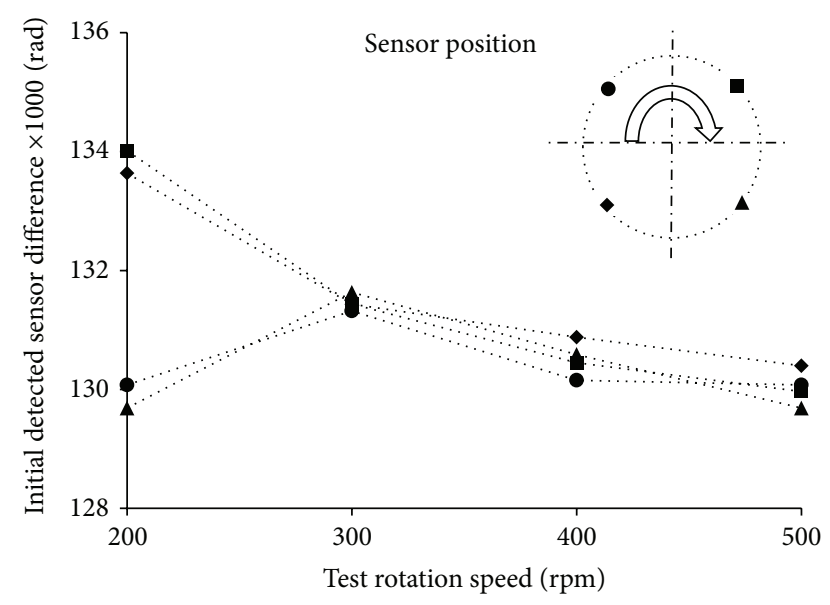

FIGURE 4: Initial angle interval of attached sensors on the shaft.

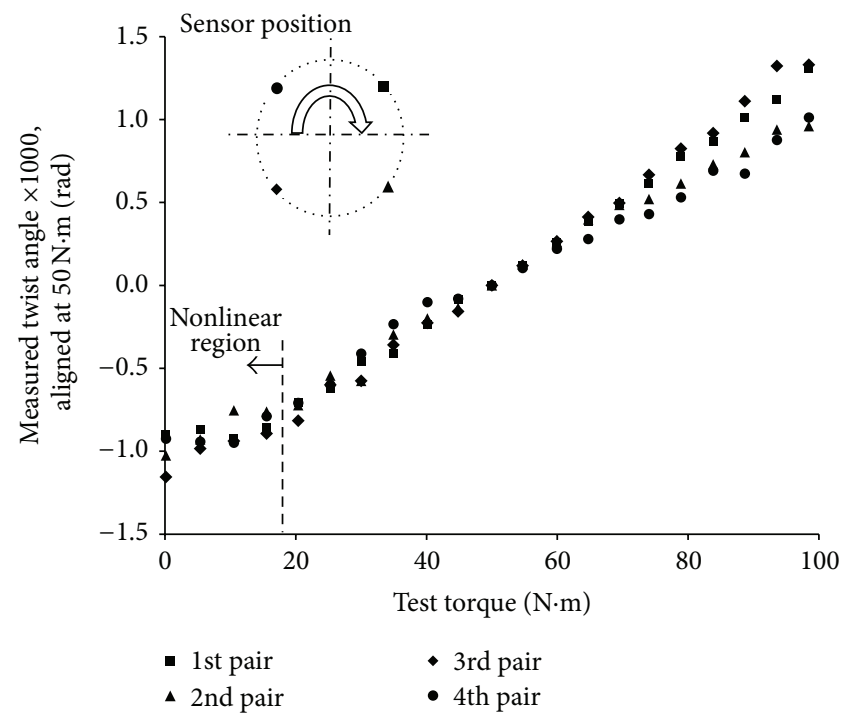

FIGURE 5: Nonlinear relationship interval at low torque (300 rpm).

in (1) is shear modulus $G$. This can be compensated with a 4thorder polynomial equation, but we ignore the temperature effect because the temperature difference is only $5^{\circ} \mathrm{C}$ during all experiments.

4.2. Speed Range for Sensor Test. Initially, the targeted speed range for the experiment was 100-1000 rpm. However, above $600 \mathrm{rpm}$, we cannot continuously increase the torque more than $100 \mathrm{~N} \cdot \mathrm{m}$ because the current test brake system only cools down by air. In addition, the chosen NI's DAQ card can operate only at $102.4 \mathrm{kHz}$ maximum. This is thought to limit the maximum shaft speed to under $600 \mathrm{rpm}$. Figure 6 shows the measured data in rotational speed versus torque per twist angle and denotes the measured twist angle with a torque increasing by $100 \mathrm{~N} \cdot \mathrm{m}$ every $5 \mathrm{~N} \cdot \mathrm{m}$. As in Figure 6, the repeated deviations and twist angle quantitative differences under $250 \mathrm{rpm}$ show very unstable results.

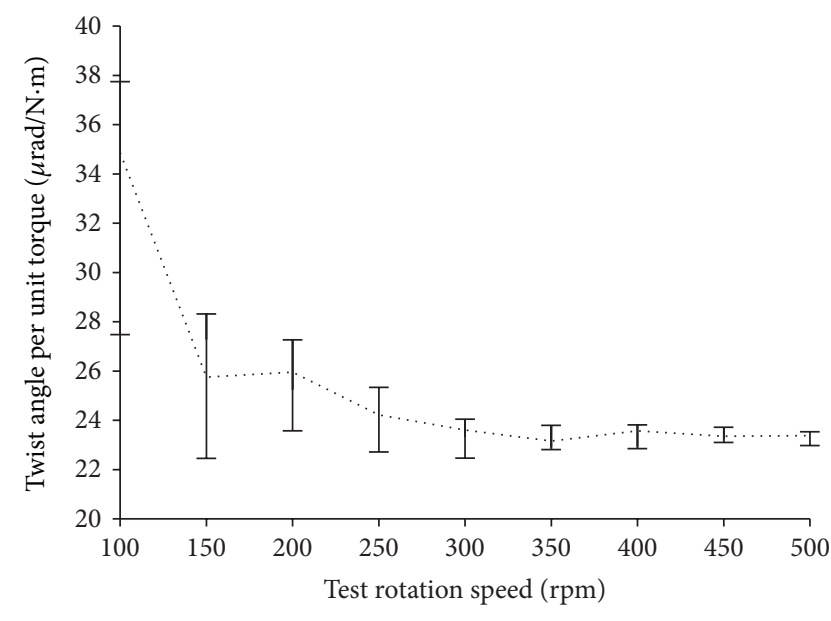

FIGURE 6: Twist angle per unit torque in the 100-500 rpm range.

These results can be explained in two ways. The first cause is the misalignment of the shafts. The coupling jointed with the torque motor, the proposed torque sensor, the commercial sensors, and the friction clutch make a comparable vibration. The other cause is a position mismatch of the new sensor and the strain-gauge-type sensor: between the two sensors are ball bearings that cause a loss in torque because the bearings exhibit different friction coefficients for a variable speed range.

For the present sensor system, the general quality of torque measurements is improved except in the low-rpm range. For better dynamic torque measurement in the present system, we isolated the torque-measuring module and shaft from outside vibration. The sensor body would move by a relative motion to maintain the distance between the magnets and the sensors.

However, we failed to obtain an ideal uniform value during testing. We observe that the cause of this failure is probably the ball-bearing gap on both sides of the modules.

Figure 7 shows average twist angles from the proposed sensor system for each applied torque in the $200-500 \mathrm{rpm}$ range. The trend lines with the least-square method almost overlap. This means the system is accurate in the 300$500 \mathrm{rpm}$ range.

4.3. Comparison of Experimental Data. In a previous approach [13] with a pair of magnets, the error was not negligible for every rotating speed that could not compensate for the shaft vibration. After installing the four pairs of magnets at $90^{\circ}$ intervals, the four cycles of sine waves can be acquired from one motor rotation cycle. The signals from each pair of magnets can compensate for the vibration of the magnet effect that is located at a $180^{\circ}$ difference. The average of the values of repeatability in the multisensor method improved significantly. Experiments in the $300-500 \mathrm{rpm}$ range were carried out with less than $2 \%$ of linear and repeatable data.

Figure 8 shows real measuring data and the average value with each pair of sensors at $400 \mathrm{rpm}$. The solid line 


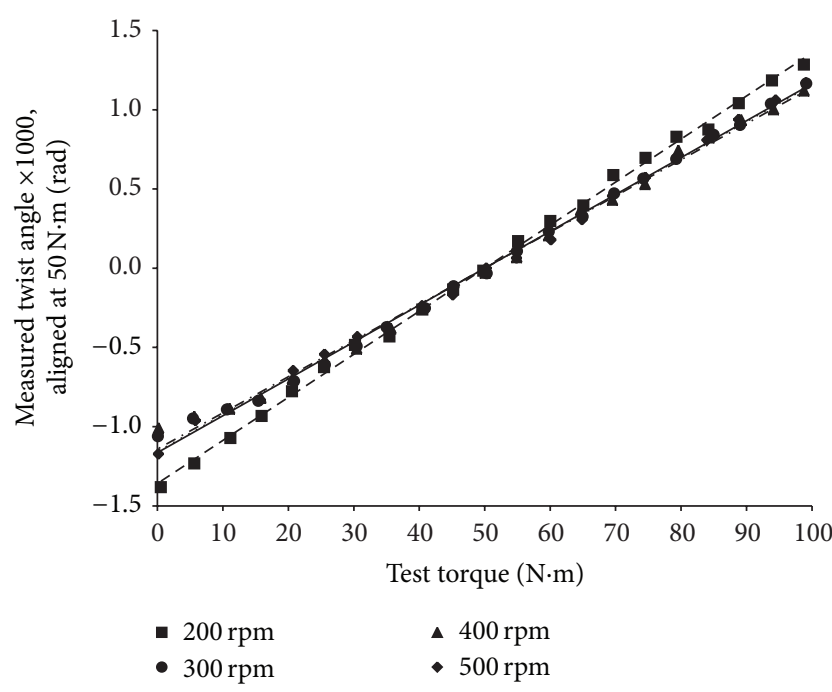

FIGURE 7: Measured average twist angle at 200-500 rpm.

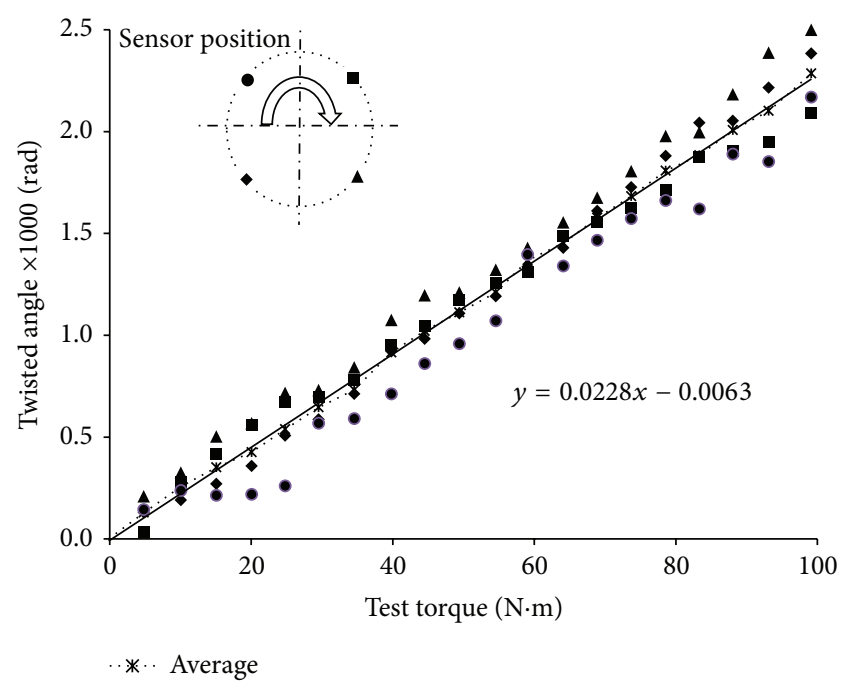

FIGURE 8: Measured twist angle of four pairs of sensors at $400 \mathrm{rpm}$.

represents the linearized average data. The signal data for each pair do not seem to exhibit linearity. However, every point $(*)$ of the average twist angle and the linearized solid line exhibit a deviation that is smaller than $2 \%$. Hence, regarding nonlinearity, we recognize that the multimagnet method is superior to the single-magnet method.

After we calibrated the new torque meter, we compared the above theoretical data in (1) and the experimental data at $400 \mathrm{rpm}$ :

$$
\begin{aligned}
\Phi & =\frac{2 L}{\pi R^{4} G} T=\frac{2 * 0.06}{\pi * 0.0125^{4} * 70 * 10^{9}} T \\
& =2.236 * 10^{-5}(\mathrm{rad} / \mathrm{N} \cdot \mathrm{m}) * T,
\end{aligned}
$$

where $L=0.06 \mathrm{~m}, R=0.0125 \mathrm{~m}$, and $G=70 \mathrm{GPa}$. The constant values $(\mathrm{rad} / \mathrm{N} \cdot \mathrm{m})$ are $99.1 \%$ and matched the slope of Figure 8 of the experimental results. Where the theoretical slope value is constant-which is currently tested with the experimental devices-the high matching ratio shows that the new torque measuring system exhibits better accuracy during experiments.

\section{Conclusion}

In this study, a noncontact-type torque sensor was evaluated to examine in variable torque condition its performance through the test bench with a rotating shaft. The shaft torque value was obtained by using four pairs of permanent neodymium magnets attached to the surface of the shaft and a pair of magnetic Hall sensors. The measured magnetic field shows the intervals between peaks. In addition, the frequency detected by the sensor helps to calculate rotational speed, which is the most important variable when converting from the time gap to a twisted angle.

The repeatability of the measured initial-angle difference at each rotational speed between magnets is less than $2 \%$. This does not appear to satisfy the needs of the automotive industry. However, we expect that the result will improve and become more accurate when we employ a higher-grade hardware system.

To guarantee the performance of the torque sensor, a calibration step or phase correction is necessary. Sufficient data regarding constraint, material properties, and geometry are required, in order to apply them to a successful torque sensor by assuming a linear deformation in the rotational direction.

Specifications for the commercial vehicle industry are needed, and future tests should include these important key points.

(i) To eliminate vibration effects, the sensor should complement any effect by the fixed part of the axis of rotation.

(ii) Measurements should be possible even at high rotational speeds. To increase the effectiveness of the hardware configuration and the algorithm for highspeed processing of data, high-speed data acquisition must be possible.

(iii) Measurements over a low-torque region need to be improved with regard to linearity, in order to obtain accurate data after modifications to the test bench and realignment shaft and after upgrading the motor or bearings.

(iv) A molding process is needed to protect the Hall sensor from oil and dust in actual conditions and to reduce the size of the entire module. To ensure that operation is free from operating temperature, we should employ the calibration and repeatability test process because the elastic modulus is temperature dependent.

\section{Conflict of Interests}

The authors declare that there is no conflict of interests regarding the publication of this paper. 


\section{Acknowledgment}

This work was supported by Inha University.

\section{References}

[1] W. J. Fleming, "Overview of automotive sensors," IEEE Sensors Journal, vol. 1, no. 4, pp. 296-308, 2001.

[2] T. Tsujisawa and K. Yamakawa, "Improvement of the angulardependent noise in a magneto-striction type torque sensor," International Journal of Automotive Engineering, vol. 2, no. 3, pp. 75-80, 2011.

[3] T. Tsujisawa and K. Yamakawa, "Proposal and performance evaluation of a magneto-striction type torque sensor consisting of small-sized coils connected in series," ISRN Electronics, vol. 2012, Article ID 738597, 8 pages, 2012.

[4] H. Wakiwaka and M. Mitamura, "New magnetostrictive type torque sensor for steering shaft," Sensors and Actuators, A: Physical, vol. 91, no. 1-2, pp. 103-106, 2001.

[5] H. Ruser, U. Troltzsch, and M. Horn, "Low-cost magnetic torque sensor principle," Sensors, vol. 2, no. 2, pp. 901-904, 2002.

[6] C. M. Cai, K. Mohri, Y. Honkura, and M. Yamamoto, "Improved pulse carrier MI effect by flash anneal of amorphous wires and FM wireless CMOS IC torque sensor," IEEE Transactions on Magnetics, vol. 37, no. 4, pp. 2038-2041, 2001.

[7] V. Kalinin, "Wireless physical SAW sensors for automotive applications," in Proceedings of the IEEE International Ultrasonics Symposium (IUS '11), pp. 212-221, October 2011.

[8] R. Xie, T. Ma, Y. Wu, and Z. Xie, "Study on torque measurement of revolving shaft based on capacitive grating sensing technology," in Proceedings of the International Conference on Electronic and Mechanical Engineering and Information Technology (EMEIT '11), vol. 9, pp. 4520-4523, August 2011.

[9] R. F. Wolffenbuttel and J. A. Foerster, "Noncontact capacitive torque sensor for use on a rotating axle," IEEE Transactions on Instrumentation and Measurement, vol. 39, no. 6, pp. 1008-1013, 1990.

[10] D. Angleviel, D. Frachon, and G. Masson, "Development of a contactless hall effect torque sensor for electric power steering," SAE Technical Paper 2006-01-0939, SAE, 2006.

[11] C. Piao, Study on wireless torque sensor by monitoring magnetic field [Ph.D. thesis], Mechanical Engineering Department, INHA University, Incheon, Korea, 2006.

[12] G. Shi, Research of a non-contact torque sensor based on magnetic field detecting [M.S. thesis], Department of Mechanical Engineering, Inha University, Incheon, Republic of Korea, 2011.

[13] G. Shi, N. Wang, and C. Cho, "Design of a new non-contact torque sensor for rotating stepped shaft by monitoring magnetic field," Applied Mechanics and Materials, vol. 44-47, pp. 547-551, 2010.

[14] J. Fraden, Handbook of Modern Sensors: Physics, Designs, and Applications, Springer, New York, NY, USA, 4th edition, 2010.

[15] T. C. Lee, "On algorithms for ordinary least squares regression spline fitting: a comparative study," Journal of Statistical Computation and Simulation, vol. 72, no. 8, pp. 647-663, 2002.

[16] J. Astola, P. Haavisto, and Y. Neuvo, "Vector median filters," Proceedings of the IEEE, vol. 78, no. 4, pp. 678-689, 1990. 

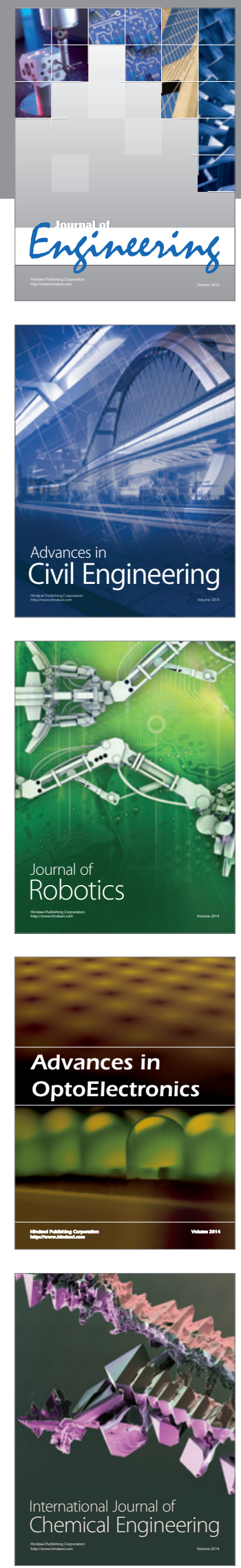

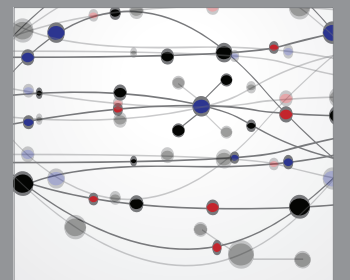

The Scientific World Journal
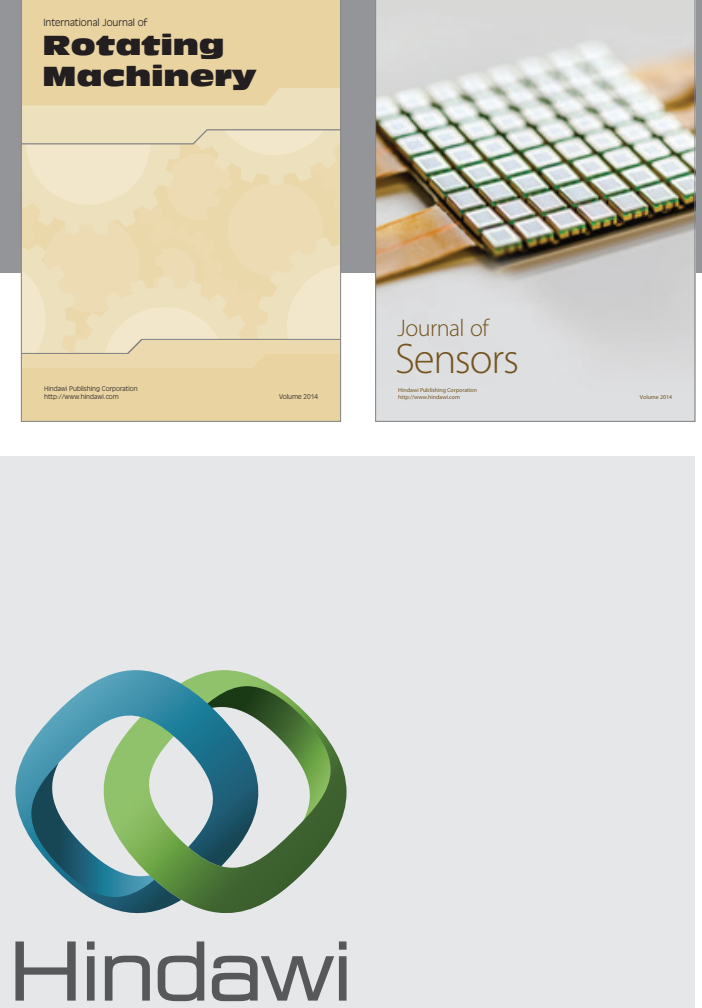

Submit your manuscripts at http://www.hindawi.com
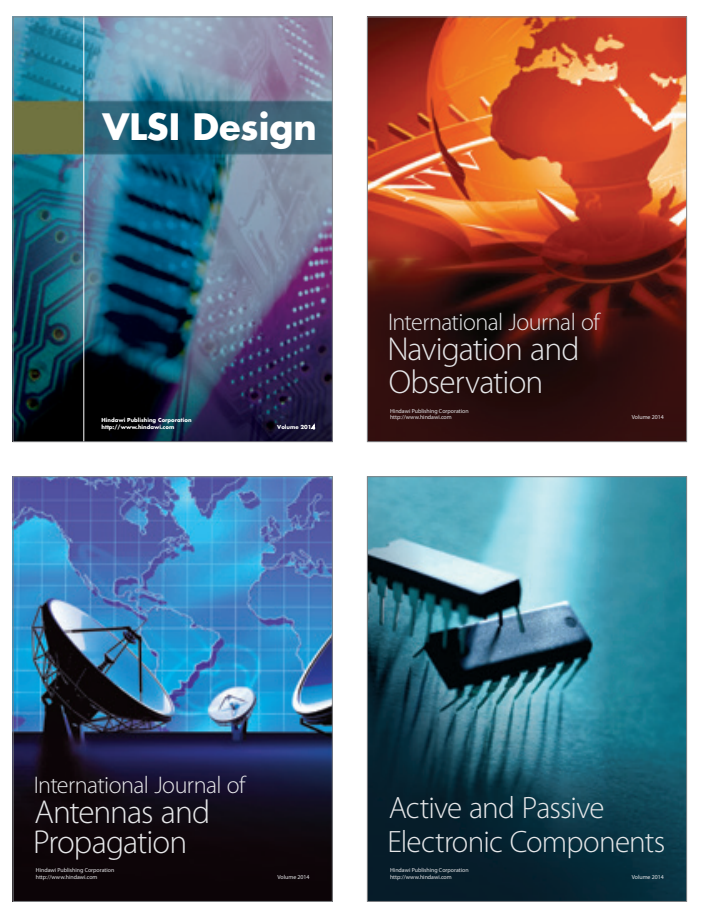
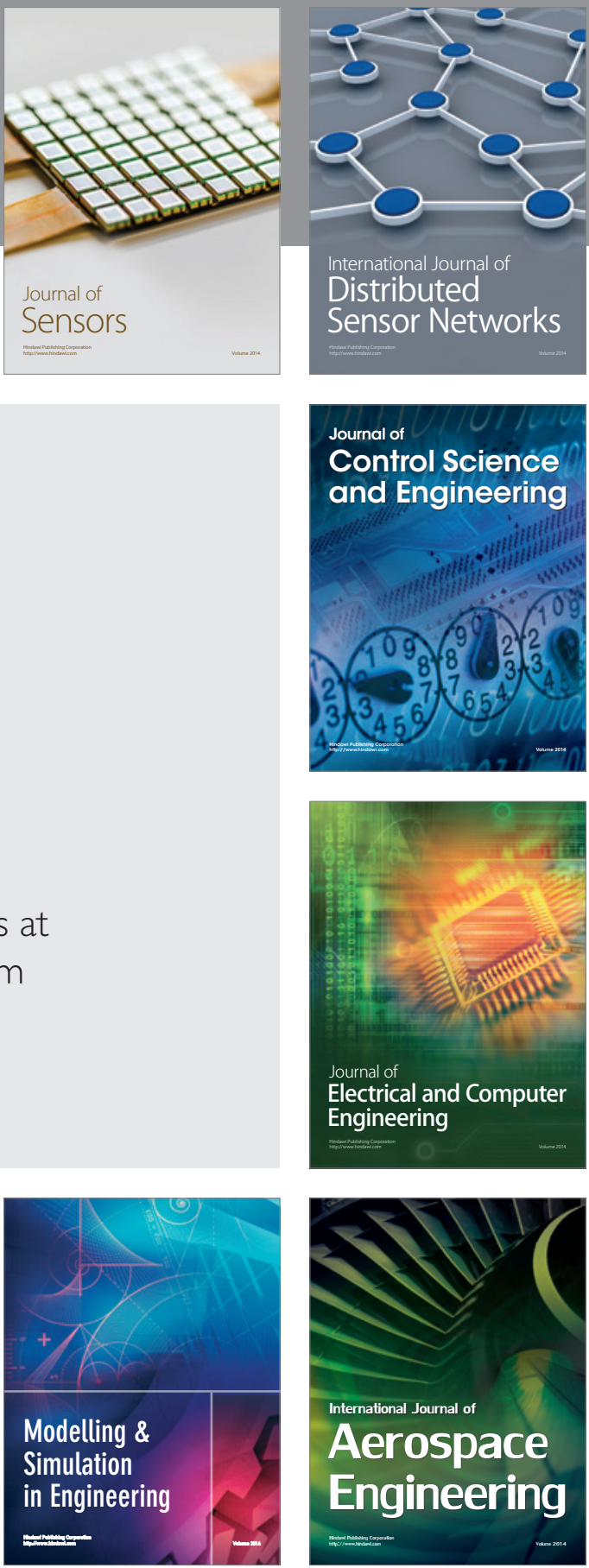

Journal of

Control Science

and Engineering
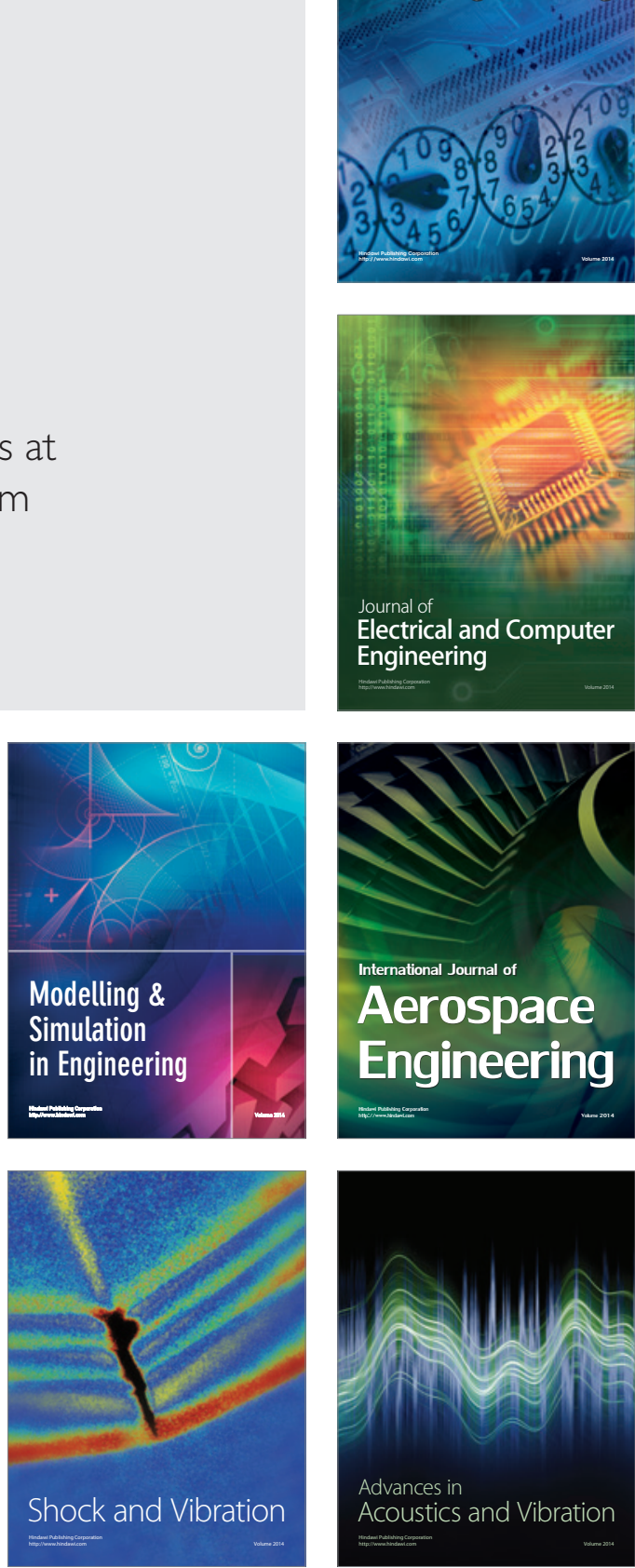